\title{
RISCOS, COMPLEXIDADE E INCERTEZAS NA CADEIA DE SUPRIMENTOS
}

uma revisão sistemática de literatura

\author{
Idoneu Mitrano Lima Junior ${ }^{1}$ \\ Centro Federal de Educação Tecnológica Celso Suckow da Fonseca \\ idoneu.junior@aluno.cefet-rj.br
}

\author{
Alba Regina Pereira Rodrigues ${ }^{2}$ \\ Centro Federal de Educação Tecnológica Celso Suckow da Fonseca \\ alba.rodrigues@cefet-rj.br \\ José André Villas Boas Mello ${ }^{3}$ \\ Centro Federal de Educação Tecnológica Celso Suckow da Fonseca \\ jose.mello@cefet-rj.br
}

\begin{abstract}
Resumo
Este artigo tem como objetivo conduzir uma revisão sistemática de literatura e meta-análise sobre o tema Supply Chain Risk Management (SCRM), no contexto dos países Brasil e China. Para tanto, os autores apoiaram-se na metodologia PRISMA (Preferred Reporting Items for Systematic reviews and Meta-Analyses), seguindo orientações da comunidade científica Cochrane Collaboration. A partir de pesquisa eletrônica nas bases Web of Science, Scopus e SciELO, os resultados open access sumarizados apontaram que há escassez de publicações sobre o tema SCRM no Brasil e na China, com apenas 6 e 8 resultados, respectivamente, no período de 2008 a 2020. Portanto, complementa as revisões sistemáticas publicadas anteriormente, auxiliando novos pesquisadores e traz como diferencial nova abordagem, evidenciando territórios sub representados nas bases analisadas.
\end{abstract}

Palavras-chave: PRISMA. Revisão Sistemática de Literatura. Gerenciamento de Riscos. Cadeia de Suprimentos.

\section{RISKS, COMPLEXITY AND UNCERTAINTIES IN THE SUPPLY CHAIN}

\section{Abstract}

a systematic literature review

This article aims to bring a systematic review of literature and meta-analysis about Supply Chain Risk Management (SCRM) in the context of Brazil and China. Therefore, the authors based themselves in PRISMA (Preferred Reporting Items for Systematic Reviews and Meta-Analysis) methodology, following Cochrane Collaboration science community orientation. After electronic researches on Web of Science, Scopus and SciELO online bases, the summarized open access results pointed to a scarcity of published topics about SCRM in Brazil and China, with 6 and 8 outcomes respectively, between 2008 and 2020. Thus, this article complements the already published systematic reviews, giving support to new researchers, and brings as its differential a new approach, putting underrepresented countries in evidence in the analyzed bases.

Keywords: PRISMA. Systematic Literature Review. Supply Chain. Risk Management.

\footnotetext{
${ }^{1}$ Pesquisador, Programa de Pós-graduação em Desenvolvimento Regional e Sistemas Produtivos, Centro Federal de Educação Tecnológica Celso Suckow da Fonseca, Campus Nova Iguaçu, Rio de Janeiro, Brasil.

2 Professora pesquisadora, Programa de Pós-graduação em Desenvolvimento Regional e Sistemas Produtivos, Centro Federal de Educação Tecnológica Celso Suckow da Fonseca, Campus Valença, Rio de Janeiro, Brasil.

${ }^{3}$ Professor pesquisador, Programa de Pós-graduação em Desenvolvimento Regional e Sistemas Produtivos, Centro Federal de Educação Tecnológica Celso Suckow da Fonseca, Campus Nova Iguaçu, Rio de Janeiro, Brasil.
} 


\section{INTRODUÇÃO}

$\mathrm{Na}$ atualidade, existem diversas lacunas no conhecimento produzido sobre Supply Chain Risk Management (SCRM), existindo divergências até mesmo na definição conceitual de tema, podendo ser vagas ou ambíguas em razão da natureza multidisciplinar e complexidade do tema (FAN ; STEVENSON, 2018a). Também há necessidade de explorar o SCRM em cadeias de suprimentos localizadas em países em desenvolvimento, que atualmente estão sub representados, resultando em escassez de estudos publicados pelas principais bases.

A revisão de literatura compõe parte do processo de elaboração de artigos, quando o pesquisador estuda as pesquisas prévias (COCHRANE e MELLO, 2020; CONCOLATO et al., 2020). No entanto, em abordagens sobre gestão de riscos em cadeias de suprimentos, muitas vezes, as pesquisas não são conduzidas por métodos estruturados de revisões de literatura, podendo levar a direções e resultados equivocados. Rodrigues et al., (2019) sugerem as revisões sistemáticas estruturadas, pois contribuem para redução das lacunas no conhecimento, uma vez que se configuram como alternativas que auxiliam na condução das pesquisas, clarificando e evidenciando os assuntos a serem explorados.

A partir disso, este artigo pretende apresentar uma revisão sistemática de literatura sobre o SCRM no contexto dos países Brasil e China, apoiando-se no diagrama da recomendação PRISMA (Preferred Reporting Items for Systematic reviews and Meta-Analyses), seguindo as orientações da comunidade científica Cochrane Collaboration. A diretriz PRISMA foi publicada em 2009, sendo resultado de uma atualização do QUOROM (QUality Of Reporting Of Meta-analyses), lançado em 2005. Essa evolução foi bem recebida por pesquisadores, resultando em alta aceitação, sobretudo pela comunidade científica (PAGE ; MOHER, 2017).

Estudar o risco em sistemas produtivos possui relevância, visto que pode influenciar o gerenciamento de um projeto e, em última instância, o desempenho organizacional (SBARDELOTTO et al., 2017). Em linhas gerais, está pesquisa conduziu uma revisão sistemática de literatura aplicando as diretrizes do PRISMA e, para tanto, está estruturada em 3 seções: a primeira seção é composta pela corrente introdução; na seção 2, é realizada uma revisão de literatura sobre os principais tópicos deste estudo, o SCRM e a recomendação PRISMA; em seguida, na seção 3, são detalhados os métodos utilizados; na seção 4, são apresentados os resultados e discussões da revisão e da meta-análise; por último, na seção 5, esta pesquisa encerra apresentando as conclusões. 


\title{
2 REVISÃO DE LITERATURA
}

A necessidade de realizar prevenções em sistemas cada vez mais complexos requer acesso a vários tipos distintos de habilidades que as pequenas e médias empresas podem não possuir (REQUEIJO et al., 2018). Na atualidade, as cadeias de suprimento vêm aumentando em complexidade e incerteza nas redes empresarias (IVANOV; DOLGUI, 2019). Componentes, insumos, produtos semiacabados e produtos finais percorrem as redes de fabricantes e consumidores de vários países no mundo (JEONG et al., 2020). Os fatores de exposição ao risco, como a terceirização, redução da base de fornecimento, just-in-time e ciclos de vida de produtos mais curtos (FAN ; STEVENSON, 2018a), trazem atenção ao tema.

O termo Gerenciamento de Riscos na Cadeia de Suprimentos pode ser compreendido como a intersecção de conhecimento entre os temas Gestão de Riscos e Gestão da Cadeia de Suprimentos (OLIVEIRA et al., 2018) e, apesar de ter surgido apenas recentemente como um campo de pesquisa distinto, são diversos os artigos publicados com o objetivo de revisar a literatura (BARYANNIS et al., 2019), muitas vezes, sem métodos de revisão estruturado como o PRISMA.

É necessário estender os estudos sobre o SCRM em diferentes contextos geográficos, uma vez que o risco nas cadeias de suprimentos estão interligados ao contexto social e às peculiaridades dos países em que se encontram, recomendando-se estudos em países em desenvolvimento (FAN; STEVENSON, 2018a, 2018b). Sobre o SCRM especificamente no contexto dos parceiros comerciais, entende-se que.

\begin{abstract}
O contexto é um elemento importante do Supply Chain Risk Management; por exemplo, a natureza do risco ou a eficácia de uma estratégia provavelmente será afetada pela indústria, país, etc. Há uma necessidade de conduzir pesquisas adicionais em contextos sub-representados, incluindo países em desenvolvimento (Fan ; Stevenson, 2018a).
\end{abstract}

Riscos podem comprometer a viabilidade dos projetos (SOUZA; DANTAS, 2020). Na literatura existem diferentes perspectivas em relação às fontes de riscos, surgindo diferentes classificações que se complementam. No presente artigo foram consideradas cinco fatores de riscos, conforme definição de Jeong, Cha e Kim (2020):

1) Risco de transporte: fatores de risco que ocorrem durante o transporte, incluindo atraso na chegada do produto ou danos ao produto durante o transporte; 
2) Risco de informação e previsão: fatores de risco relacionados a informações e previsão de demanda, incluindo erros relacionados a pedidos (documentação de pedidos, entrada de pedidos e erros do sistema de informações), solicitações urgentes de pedidos e alterações por clientes e manutenção adequada de estoque.

3) Risco do fornecedor: fatores de risco relacionados aos fornecedores no SCM, incluindo falência repentina do fornecedor, escassez e defeitos causados pela capacidade de produção inadequada do fornecedor e a incapacidade de responder a pedidos urgentes.

4) Risco ambiental: mudanças no ambiente operacional do SCM, incluindo flutuações de preços devido ao aumento dos preços das matérias-primas e inflação; oferta e demanda não atendidas devido à insuficiência de matérias-primas; mudanças nos regulamentos, leis e políticas relacionadas; e mudanças tecnológicas.

5) Risco destrutivo: desastres e acidentes repentinos, incluindo desastres naturais inesperados, epidemias, incêndios, acidentes de trânsito, greves e fechamentos de portos e aeroportos.

\section{MÉTODO}

Uma revisão sistemática de literatura estruturada deve utilizar métodos sistemáticos e explícitos para identificar, selecionar e avaliar criticamente as pesquisas, enquanto a metaanálise se refere as técnicas estatísticas utilizadas na análise dos estudos que foram incluídos na revisão. A recomendação PRISMA foi elaborada com o objetivo de abranger tanto as revisões sistemáticas quanto as meta-análises, contando com um checklist com vinte e sete itens, um fluxograma de quatro etapas (MOHER et al., 2009) e quinze extensões, sendo sete publicadas (conforme Quadro 1) e oito em desenvolvimento (PAGE; MOHER, 2017).

Quadro 1- Escopo da declaração prisma e extensões publicadas até 2017

\begin{tabular}{|c|c|l|}
\hline $\begin{array}{c}\text { Relatórios de } \\
\text { Diretrizes }\end{array}$ & $\begin{array}{c}\text { Ano de } \\
\text { Publicação }\end{array}$ & \multicolumn{1}{c|}{ Escopo dos Relatórios } \\
\hline PRISMA & 2009 & $\begin{array}{l}\text { Relatórios de revisões sistemáticas e meta-análises, principalmente de } \\
\text { ensaios clínicos randomizados que avaliam intervenções de cuidados de } \\
\text { saúde. }\end{array}$ \\
\hline $\begin{array}{c}\text { PRISMA } \\
\text { Equity }\end{array}$ & 2012 & $\begin{array}{l}\text { Relatórios de revisões sistemáticas e meta-análises com foco na equidade } \\
\text { em saúde, definida como ausência de desigualdades evitáveis e injustas em } \\
\text { saúde. }\end{array}$ \\
\hline $\begin{array}{c}\text { PRISMA } \\
\text { Abstracts }\end{array}$ & $\begin{array}{l}\text { Resumos para todos os tipos de revisões sistemáticas, mas a ênfase está em } \\
\text { revisões sistemáticas de avaliações de intervenções onde uma ou mais } \\
\text { meta-análises são conduzidas. }\end{array}$ \\
\hline
\end{tabular}


INOVAÇÃO

\begin{tabular}{|c|c|c|}
\hline $\begin{array}{c}\text { PRISMA } \\
\text { Network Meta } \\
\text { - Analysis }\end{array}$ & 2015 & $\begin{array}{l}\text { Relatórios de revisões sistemáticas que abordam redes de comparações de } \\
\text { múltiplos tratamentos. }\end{array}$ \\
\hline $\begin{array}{c}\text { PRISMA } \\
\text { Individual } \\
\text { Participant } \\
\text { Data }\end{array}$ & 2015 & $\begin{array}{l}\text { Relatórios de revisões sistemáticas e meta-análises de dados de } \\
\text { participantes individuais. Desenvolvido principalmente para revisões de } \\
\text { ensaios clínicos randomizados, mas muitos itens se aplicam a outros } \\
\text { contextos, incluindo revisões de diagnóstico e prognóstico. }\end{array}$ \\
\hline $\begin{array}{c}\text { PRISMA } \\
\text { Protocols }\end{array}$ & 2015 & $\begin{array}{l}\text { Protocolos para revisões sistemáticas e meta-análises que } \\
\text { resumem dados agregados de estudos, particularmente aqueles que avaliam } \\
\text { os efeitos das intervenções. }\end{array}$ \\
\hline $\begin{array}{c}\text { PRISMA } \\
\text { Harms }\end{array}$ & 2016 & $\begin{array}{l}\text { Relatórios de revisões sistemáticas e meta-análises avaliando eventos } \\
\text { adversos (como um primário ou resultado secundário) que são relatados em } \\
\text { estudos prospectivos de intervenção ou estudos observacionais (com ou } \\
\text { sem grupo de comparação) }\end{array}$ \\
\hline $\begin{array}{c}\text { PRISMA } \\
\text { Complex } \\
\text { Interventions }\end{array}$ & 2017 & $\begin{array}{l}\text { Relatórios de revisões sistemáticas e meta-análises de intervenções } \\
\text { complexas. Intervenções complexas são definidos como intervenções que } \\
\text { têm componentes múltiplos (complexidade de intervenção) e vias causais } \\
\text { complicadas/múltiplas, ciclos de feedback, sinergias e/ou mediadores e } \\
\text { moderadores do efeito (complexidade da via). }\end{array}$ \\
\hline
\end{tabular}

Fonte: adaptado de Page e Moher (2017).

Dentre as questões conceituais na evolução do QUORUM para o PRISMA, segundo Moher et al. (2009), é possível ressaltar: 1) a revisão sistemática como processo interativo, podendo ser modificado o protocolo de revisão original. 2) a distinção conceitual entre conduta versus relato de pesquisa; 3) a avaliação meticulosa do risco de viés nos estudos incluídos; e 4) a importância de relatar os vieses. Sobre o critério avaliação dos vieses de publicação, embora sua ausência não implique necessariamente que não tenha sido realizado, é provavelmente o marcador do rigor na conduta da revisão sistemática. Deste modo, a declaração PRISMA pode ser útil para, além de conduzir novas revisões, também avaliar a técnica empregada em revisões sistemáticas já publicadas (MOHER et al., 2009).

Em relação às revisões sistemáticas disponíveis, destaca-se o estudo de Fan e Steverson (2018a), que adotou um método de revisão de literatura baseado em sete etapas. Por outro lado, Oliveira et al. (2018) utilizaram a bibliometria e mineração de texto para o desenvolvimento de um perfil de pesquisa, denominado por Porter et al. (2002) como Research Profiling. Enquanto Baryannis et al. (2019), descreve que sua estratégia de pesquisa se baseou-se principalmente na pesquisa automatizada, utilizando dois níveis de palavra-chave nas buscas nas principais bases, como é sugerido pelo estudo de Prakash, Soni e Rathore (2017).

Os métodos empregados por estes autores não contemplam (explicitamente) todos os itens listados na recomendação PRISMA e, desta forma, visando complementar as pesquisas anteriores, argumenta-se que se faz necessária uma nova e rigorosa pesquisa que adote a recomendação PRISMA como método (explicitamente). Porém, vale ressaltar que o PRISMA 
não pode ser utilizado como instrumento de avaliação da qualidade das diversas revisões sistemáticas publicadas (MOHER et al, 2009), pois os pesquisadores podem ter conduzido a revisão de literatura adotando todos os critérios, ainda que não esteja explícito ou relatado.

De acordo com fluxograma do PRISMA, buscou-se cumprir as etapas de identificação, seleção, elegibilidade e inclusão dos artigos, conforme Figura 1. Os autores dos artigos incluídos não foram contatados e os gráficos propostos na meta-análise foram gerados a partir do Microsoft Excel.

Figura 1- fluxograma PRISMA para inclusão de artigos

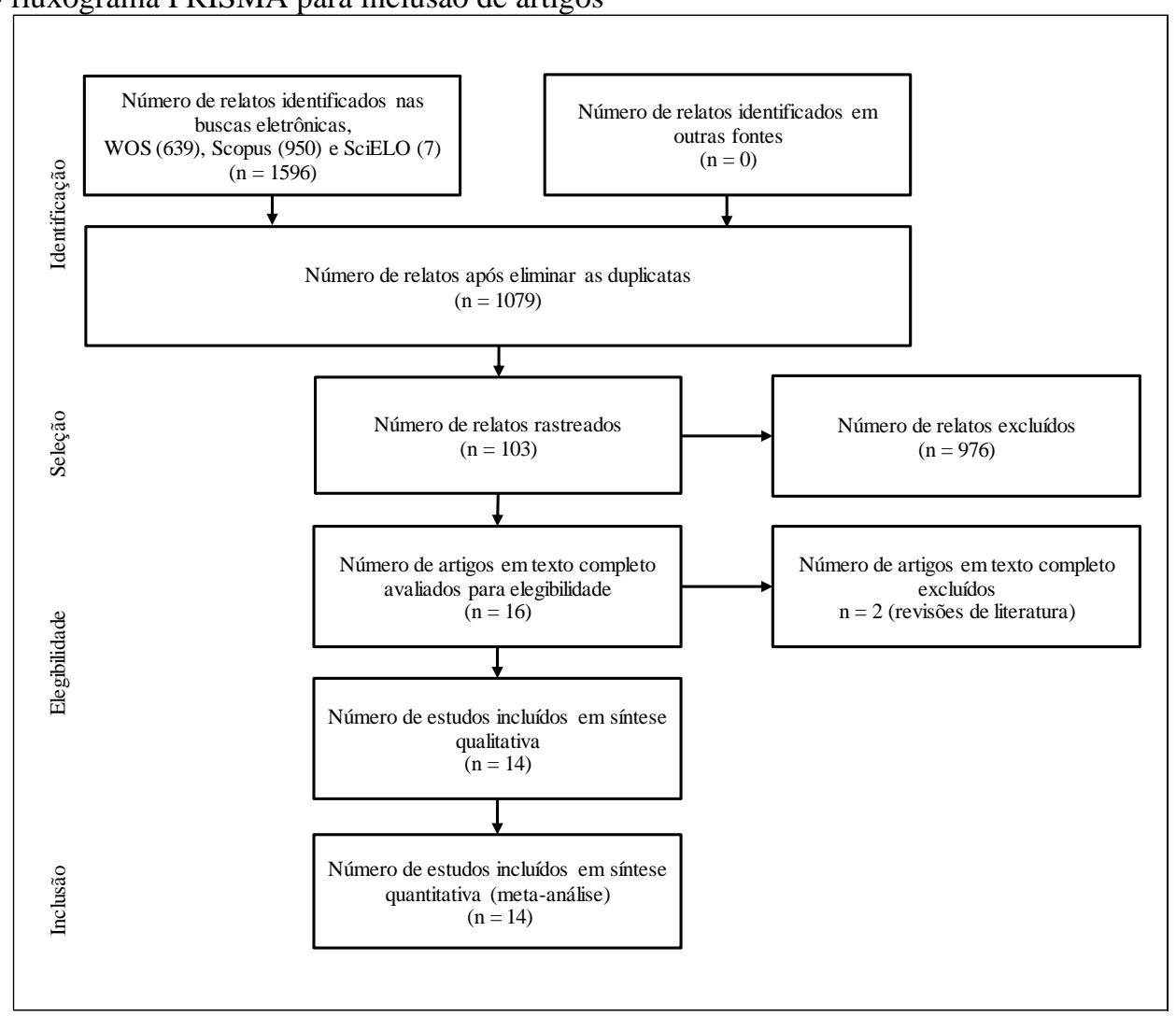

Fonte: Autores

\section{Passo 1: identificação}

Na etapa de identificação, o termo utilizado nas buscas foi "Supply Chain Risk Management". A data de realização das buscas foi entre 01/10/2020 e 15/10/2020. Foram identificados 1596 artigos, distribuídos em 639 artigos na base Web of Science, 950 na Scopus e 7 na SciELO. Em seguida, após a eliminação de trabalhos duplicados, foram reduzidos a 1079 artigos no total.

A justificava para escolha das bases para as buscas eletrônicas foram: Web of Science (Clarivate Analytics) e Scopus (Elsevier) por serem os maiores serviços de indexação na 
atualidade (MONGEON ; PAUL-HUS, 2016), concentrando a maior parte das publicações sobre o tema Supply Chain Risk Management (OLIVEIRA et al., 2018). A SciELO foi escolhida pela evidente representatividade dos pesquisadores brasileiros: segundo a SciELO Analyties (2020), o Brasil lidera o ranking mundial em número de publicações por país. Assim, as três bases são suficientes para a proposta desta pesquisa. Este trabalho não utilizou busca manual.

\section{Passo 2: seleção}

Visando obter o maior número de publicações, somente dois critérios de inclusão foram adotados: 1) as publicações devem estar disponibilizadas como "acesso aberto", alinhado ao crescente movimento mundial de políticas de acesso aberto à pesquisa (Xavier, 2019); 2) as pesquisas devem ser classificadas como "artigos" revisados por pares, em razão da natureza deste artigo, nas revisões sistemáticas recomenda-se analisar somente este tipo de documento.

Todos os demais critérios não foram alterados, seguindo em default, como por exemplo: o "ano da publicação" permaneceu inalterado, permitindo a busca de artigos a partir de 1945 (WOS). Assim como o "idioma", ainda que a maior parte dos artigos sejam elaboradoras na língua inglesa, este não foi um critério de exclusão desta pesquisa.

$\mathrm{Na}$ etapa de seleção, 976 pesquisas foram excluídas, restando 103 publicações para a próxima etapa.

\section{Passo 3: elegibilidade}

Nesta etapa, como critério de elegibilidade, buscou-se artigos que discorram sobre o SCRM no contexto do território brasileiro e chinês, logo os artigos foram submetidos a análise parcial de dados, sendo avaliados os títulos, palavras-chave, resumos e país de origem. Após a triagem, 16 artigos selecionados seguiram para a análise integral de texto. Foram excluídos 2 artigos (OLIVEIRA et al., 2018; FAGUNDES et al., 2020) que foram classificados no presente estudo como revisões de literatura. Os demais (14 artigos) atenderam aos critérios de elegibilidade.

\section{Passo 4: inclusão}

Por fim, 14 artigos foram incluídos na síntese qualitativa e na síntese quantitativa. $\mathrm{Na}$ recomendação PRISMA não há regra em relação a quantidade mínima ou máxima de artigos a serem incluídos. Segundo Moher et al. (2009): a equipe de revisão deve pesquisar a literatura disponível. Esta busca resulta no número de relatos encontrados. Uma vez que estes relatos foram rastreados e os critérios de elegibilidade aplicados, um número menor de artigos remanescerá. 


\section{RESULTADOS E DISCUSSÕES}

Nesta seção, são evidenciados os resultados da meta-análise dos artigos, sumarizados em quadros, tabelas e gráficos. O período analisado corresponde a intervalo 2008-2020. Entre os periódicos que mais publicaram artigos sobre SCRM, temos empatados o "Gestão e Produção" para o contexto Brasil e o "International Journal of Production Economics" para o contexto China, com 2 registros cada, conforme Quadro 2.

Quadro 2 - publicações por periódicos

\begin{tabular}{|c|l|c|c|}
\hline Ordem & \multicolumn{1}{|c|}{ Periódico } & Registros & Contexto \\
\hline 1 & Gestão e Produção & 2 & Brasil \\
\cline { 2 - 4 } & International Journal of Production Economics & 2 & China \\
\hline 2 & Asian Journal of Shipping and Logistics & 1 & China \\
\cline { 2 - 4 } & Discrete Dynamics in Nature and Society & 1 & China \\
\cline { 2 - 4 } & Food Control & 1 & China \\
\cline { 2 - 4 } & International Food and Agribusiness Management Review & 1 & Brasil \\
\cline { 2 - 4 } & Journal of Engineering - JOE & 1 & Brasil \\
\cline { 2 - 4 } & Journal of Operations and Supply Chain Management & 1 & Brasil \\
\cline { 2 - 4 } & Rege: Revista de Gestão & 1 & Brasil \\
\cline { 2 - 4 } & Risk Management: an Internacional Journal & 1 & China \\
\cline { 2 - 4 } & Supply Chain Management - an International Journal & 1 & 1 \\
\cline { 2 - 4 } & Sustainability & & 1 \\
\hline
\end{tabular}

O tema SCRM é relativamente novo e vem ganhando notoriedade no cenário internacional. Conforme o Gráfico 1, o primeiro artigo que traz contribuições sobre o SCRM no contexto China foi publicado em 2008. Para o contexto Brasil, a publicação ocorreu somente em 2016. Há um intervalo entre os anos 2009-2015 sem quaisquer publicações. O ano de maior expressão foi 2018, com 4 artigos publicados no total, sendo 3 no contexto China. No período analisado (2008-2020), no contexto Brasil, a média de publicações foi de 0,46 artigos ao ano e o desvio-padrão foi de 0,88 . Na china, a média de publicações foi maior, de 0,62 artigos ao ano e o desvio-padrão de 1,12. No total, no período (2008-2020), analisando ambos os contextos (Brasil e China), a média de publicações de artigos por ano foi de 1,08 e o desvio-padrão 2. 
Gráfico 1 - frequência de publicações por ano

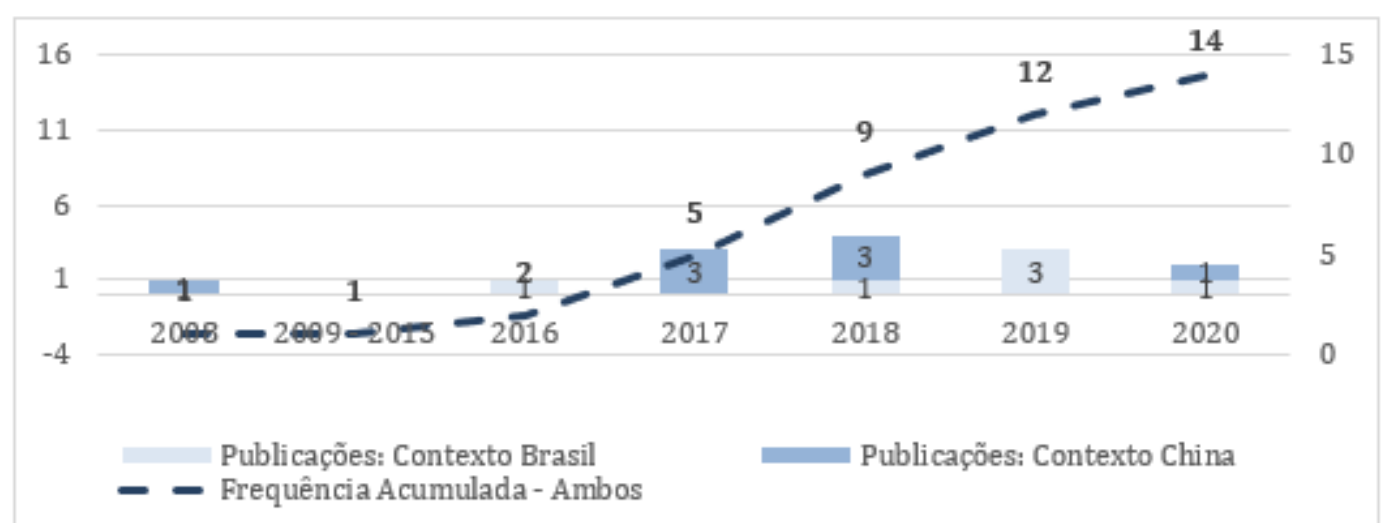

Fonte: Autores

No quadro 3, é possível constatar que as palavras-chave mais utilizadas pelos autores foram "Supply Chain Risk Management” (7 registros), "Risk Management” (4), "Supply Chain" (3) e "SCRM" (2). Ocupando a quinta posição na classificação, temos um empate entre outras 39 palavras-chave. Nos artigos de Zhou e Xun (2018) e Ji; Qu e Yu (2017) não foram identificadas as palavras-chaves.

Quadro 3 - repetição de palavras-chave

\begin{tabular}{|l|c|c|}
\hline \multicolumn{1}{|c|}{ Palavra Chave } & Registros & Contexto \\
\hline Supply Chain Risk Management & 7 & Brasil (4) e China (3) \\
\hline Risk Management & 3 & Brasil (1) e China (3) \\
\hline Supply Chain & 2 & Brasil (2) e China (1) \\
\hline SCRM & 1 & Brasil (2) \\
\hline AHP & 1 & Brasil \\
\hline Analytical Orientation & 1 & Brasil \\
\hline Analytical Supply Chains & 1 & Brasil \\
\hline Attitude & 1 & China \\
\hline Awareness Analysis & 1 & China \\
\hline Bidirectional Option Contracts & 1 & Brasil \\
\hline Brazilian Amazon & 1 & China \\
\hline Case Studies & 1 & Brasil \\
\hline Cattle & 1 & Brasil \\
\hline Cluster Analysis & 1 & China \\
\hline Coordination & 1 & China \\
\hline Decision Analysis & 1 & Brasil \\
\hline Expectation Confirmation Theory & 1 & \\
\hline Flexibility & 1 & \\
\hline
\end{tabular}




\begin{tabular}{|l|l|l|}
\hline Food Supply Chain & 1 & China \\
\hline Game Theory & 1 & China \\
\hline Hybrid Production System & 1 & Brasil \\
\hline Logistics & 1 & Brasil \\
\hline Monte Carlo Simulation & 1 & China \\
\hline MTS-MTO & 1 & Brasil \\
\hline Multiple Criteria & 1 & China \\
\hline Regulation & 1 & China \\
\hline Reputational Risk & 1 & Brasil \\
\hline Risk Awareness & 1 & China \\
\hline Risk Factors & 1 & China \\
\hline Risks & 1 & Brasil \\
\hline Seaports & 1 & Brasil \\
\hline Service Requirement & 1 & China \\
\hline Shipbuilding and Marine Equipment Market & 1 & China \\
\hline Social capital & 1 & China \\
\hline Structural Equation Modeling & 1 & Brasil \\
\hline Supplier-Manufacturer Relationships & 1 & China \\
\hline Supply Chain Risk & 1 & China \\
\hline Supply Risk & 1 & China \\
\hline Survey & 1 & Brasil \\
\hline Sustainability & 1 & Brasil \\
\hline Uncertainty & 1 & Brasil \\
\hline Value & 1 & China \\
\hline Vendor Selection & 1 & \\
\hline
\end{tabular}

Fonte: Autores

O quadro 4 visa apresentar, de forma resumida e objetiva, a pesquisa desenvolvida dos artigos analisados em texto integral. Os artigos revisados estão inseridos no cenário dos territórios escolhidos (Brasil e China) e estão listados em ordem cronológica.

Quadro 4 - pesquisas desenvolvidas por artigo

\begin{tabular}{|c|l|}
\hline \multicolumn{1}{|c|}{ Artigo } & \multicolumn{1}{c|}{ Pesquisa Desenvolvida } \\
\hline (Wu ; Olson, 2008) & $\begin{array}{l}\text { Analisam os resultados comparativos entre modelos de simulação e outros três } \\
\text { modelos para avaliação de riscos na cadeia de suprimentos. }\end{array}$ \\
\hline
\end{tabular}




\begin{tabular}{|c|c|}
\hline (Trkman et al., 2016) & $\begin{array}{l}\text { Investiga o nível de atenção e maturidade das empresas em relação ao SCRM, } \\
\text { utilizando a teoria de confirmação de expectativas. Uma parte da coleta de } \\
\text { dados é composta por } 6 \text { mini estudos de casos de empresas localizadas no } \\
\text { Canadá, EUA e Brasil. }\end{array}$ \\
\hline (Chen et al., 2017) & $\begin{array}{l}\text { Examina o impacto no nível de serviço em contratos com opção bidirecional } \\
\text { em uma cadeia de suprimento de dois escalões, consistindo em um fornecedor } \\
\text { e um varejista, levando em consideração uma exigência de serviço. }\end{array}$ \\
\hline (Ji et al., 2017) & $\begin{array}{l}\text { Sugerem um novo "modelo de programa multiobjeto de dois níveis ponderados } \\
\text { de pior caso" que pode ser útil para a estão de riscos da cadeia de suprimentos } \\
\text { com incerteza de demanda. }\end{array}$ \\
\hline (Song ; Zhuang, 2017) & $\begin{array}{l}\text { Propõe um modelo Governo-Fabricante-Fazendeiro para controle de aditivos } \\
\text { químicos utilizados na agricultura }\end{array}$ \\
\hline (Alvarenga et al., 2018) & $\begin{array}{l}\text { Verifica o impacto da orientação analítica sobre a gestão de risco da cadeia de } \\
\text { suprimentos em micro, pequenas e médias empresas da região sudeste do } \\
\text { Brasil. }\end{array}$ \\
\hline (Fan ; Stevenson, 2018b) & $\begin{array}{l}\text { Investiga como os riscos da cadeia de suprimentos podem ser identificados em } \\
\text { relacionamentos entre comprador e fornecedor colaborativos e adversários }\end{array}$ \\
\hline (Jiang et al., 2018) & $\begin{array}{l}\text { Avalia o risco da cadeia de suprimentos das empresas portuárias de Qingdao, } \\
\text { na China, através de um método AHP. }\end{array}$ \\
\hline (Zhou; Xun, 2018) & $\begin{array}{l}\text { Investiga a tomada de decisão multicritério para a seleção de fornecedores, } \\
\text { propondo um método para seleção de fornecedores baseado no processo } \\
\text { analítico de redes fuzzy, em um estudo de caso da indústria eletrônica. }\end{array}$ \\
\hline (Dias et al., 2019) & $\begin{array}{l}\text { Busca identificar quais riscos o segundo maior porto brasileiro pode oferecer } \\
\text { às organizações de sua rede, bem como eles são tratados pelos gestores do } \\
\text { porto. }\end{array}$ \\
\hline (Galuchi et al., 2019) & $\begin{array}{l}\text { Identifica as principais fontes de riscos reputacionais nas cadeias produtivas de } \\
\text { carne bovina da Amazônia brasileira e as ações tomadas pelos frigoríficos para } \\
\text { gerenciar esses tipos de risco. }\end{array}$ \\
\hline (Wolfshorndl et al., 2019) & $\begin{array}{l}\text { Aborda os efeitos de um sistema de produção híbrido (produção para estoque e } \\
\text { produção sob encomenda), a fim de saber quais riscos podem impactar o } \\
\text { processo de planejamento da produção em uma grande montadora de veículos } \\
\text { no Brasil. }\end{array}$ \\
\hline (Dias et al., 2020) & $\begin{array}{l}\text { Analisa como uma montadora brasileira identifica e gerencia os fatores de risco } \\
\text { em sua cadeia de suprimentos. }\end{array}$ \\
\hline (Jeong et al., 2020) & $\begin{array}{l}\text { Analisa as relações entre os níveis de conscientização e gerenciamento de risco } \\
\text { da cadeia de suprimentos (SCRM), o nível de desenvolvimento e sua influência } \\
\text { no desempenho dos negócios na indústria de construção naval e equipamentos } \\
\text { marítimos. Além disso, destaca as diferenças nos níveis de conscientização de } \\
\text { acordo com as características específicas do país, comparando a Coreia do Sul, } \\
\text { China e Cingapura. }\end{array}$ \\
\hline
\end{tabular}

Fonte: Autores

O quadro 5 concentra as recomendações de estudos futuros. A maior parte dos pesquisadores (71\%) declararam explicitamente as recomendações de estudos futuros, entretanto, em alguns casos, o relato não foi identificado (JI et al., 2017; JIANG et al., 2018; TRKMAN et al., 2016; WU ; OLSON, 2008). 
Quadro 5 - recomendações de estudos futuros por artigo

\begin{tabular}{|c|c|}
\hline Artigo & Recomendações de Estudos Futuros \\
\hline (Wu ; Olson, 2008) & Não identificado. \\
\hline (Trkman et al., 2016) & Não identificado. \\
\hline (Ji et al., 2017) & Não identificado. \\
\hline (Chen et al., 2017) & $\begin{array}{l}\text { Explorar o efeito de diferentes tipos de contratos de opção na cadeia de suprimentos e } \\
\text { considerar que tipo de opção contratos é a melhor escolha quando há um requisito de } \\
\text { serviço. Considerar diferentes atitudes de risco nos membros, como fornecedor ou } \\
\text { varejista avesso à perda. Considerar diferentes estruturas de energia da cadeia de } \\
\text { suprimentos e posições dominantes. Considerar parâmetros separados para o exercício } \\
\text { de opções bidirecionais como opções de compra ou venda. }\end{array}$ \\
\hline $\begin{array}{l}\text { (Song } ; \text { Zhuang, } \\
2017)\end{array}$ & $\begin{array}{l}\text { Considerar a política fiscal do governo como uma variável de decisão que afeta os } \\
\text { comportamentos de risco dos fabricantes e agricultores. Modelar comportamentos não. } \\
\text { Analisar as competições. Considerar os diferentes efeitos de produtos químicos } \\
\text { aditivos na taxa de perecimento de alimentos em um modelo dinâmico. }\end{array}$ \\
\hline \begin{tabular}{lll|} 
(Alvarenga et $a l .$, & \\
2018)
\end{tabular} & $\begin{array}{l}\text { Verificar o papel da orientação analítica na relação entre o gerenciamento de risco e } \\
\text { outras capacidades que afetam o construto, e se a orientação analítica e o } \\
\text { gerenciamento de risco ajudam as cadeias de suprimentos a se recuperar de eventos } \\
\text { inesperados, melhorando a resiliência. }\end{array}$ \\
\hline $\begin{array}{l}\text { (Fan ; Stevenson, } \\
\text { 2018b) }\end{array}$ & $\begin{array}{l}\text { Explorar outros contextos e incluir a perspectiva do fornecedor (a coleta de dados do } \\
\text { artigo é focada na China e sob perspectiva do comprador). }\end{array}$ \\
\hline (Jiang et al., 2018) & Não identificado. \\
\hline (Zhou ; Xun, 2018) & $\begin{array}{l}\text { Integrar a análise de especialistas de sistemas de computação para cálculos mais } \\
\text { refinados e meticulosos, além de obter os resultados com mais rapidez e precisão. }\end{array}$ \\
\hline (Dias et al., 2019) & $\begin{array}{l}\text { Investigar a viabilidade de estruturação do SCRM no processo de gestão nos portos } \\
\text { brasileiros, analisando as demais empresas que compõem a rede e os pontos de vista } \\
\text { de outras partes interessadas. }\end{array}$ \\
\hline (Galuchi et al., 2019) & $\begin{array}{l}\text { A condução de múltiplos estudos de caso utilizando os métodos propostos de gestão } \\
\text { de risco socioambiental em cadeias de suprimentos, em diferentes cadeias } \\
\text { agroalimentares e em diferentes circunstâncias. Ou a realização de entrevistas com } \\
\text { gerentes e partes interessadas da empresa para agregar mais elementos nas descobertas. }\end{array}$ \\
\hline $\begin{array}{l}\text { (Wolfshorndl et al., } \\
\text { 2019) }\end{array}$ & $\begin{array}{l}\text { O estudo pode ser ampliado para outras empresas que adotem ou mesmo que adotem } \\
\text { um sistema híbrido de produção. Ou pode explorar riscos funcionais decorrentes da } \\
\text { própria gestão, ou riscos restritos ao SCRM, incluindo sua relevância. Até mesmo, a } \\
\text { forma como as organizações se esforça para controlar os riscos desnecessariamente. }\end{array}$ \\
\hline (Dias et al., 2020) & $\begin{array}{l}\text { Analisar as demais empresas do cluster automotivo, de forma a obter resultados mais } \\
\text { gerais aplicáveis àquele setor. Ou analisar outros setores para fins de comparação. }\end{array}$ \\
\hline (Jeong et al., 2020) & $\begin{array}{l}\text { Aumentar a precisão dos resultados ao coletar um número semelhante de questionários } \\
\text { de cada país. Ajuste mais preciso do viés potencial decorrente do uso de dados } \\
\text { subjetivos (respostas de julgamento). Investigar o tema ampliando o escopo dos países } \\
\text { pesquisados para a Europa, Estados Unidos e outros. }\end{array}$ \\
\hline
\end{tabular}

Fonte: Autores

Os autores revisados neste artigo abordam diferentes fatores de risco em seus estudos, com diferentes denominações. Na presente pesquisa, cinco fatores de risco foram considerados: risco de transporte, risco de informação e previsão, risco de fornecedor, risco ambiental e risco destrutivo (JEONG et al., 2020). A Tabela 1 descreve quais fatores de riscos são abordados em cada artigo revisado, sendo os riscos de informação e os relativos aos fornecedores os mais 
intensos e mais estudados. As definições e características de cada fator de risco foram detalhadas na seção 2 (revisão de literatura).

Tabela 1 - fatores de riscos abordados nos artigos revisados

\begin{tabular}{|c|c|c|c|c|c|}
\hline Artigo & 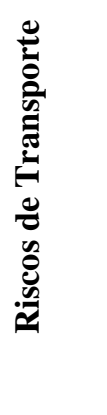 & 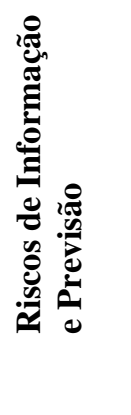 & 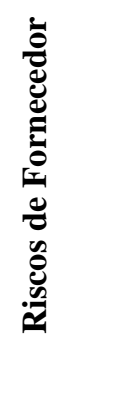 & 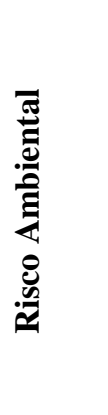 & 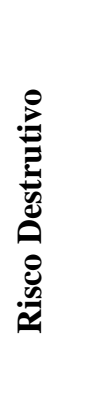 \\
\hline (Wu ; Olson, 2008) & $\mathrm{X}$ & & $\mathrm{X}$ & & \\
\hline (Trkman et al., 2016) & & $\mathrm{X}$ & & & \\
\hline (Chen et al., 2017) & & & $\mathrm{X}$ & $\mathrm{X}$ & \\
\hline (Ji et al., 2017) & & $\mathrm{X}$ & $\mathrm{X}$ & & \\
\hline (Song ; Zhuang, 2017) & & & & $\mathrm{X}$ & \\
\hline (Alvarenga et al., 2018) & & $\mathrm{X}$ & $\mathrm{X}$ & & \\
\hline (Fan ; Stevenson, 2018b) & & $\mathrm{X}$ & $\mathrm{X}$ & & \\
\hline (Jiang et al., 2018) & $\mathrm{X}$ & $\mathrm{X}$ & $X$ & $\mathrm{X}$ & $\mathrm{X}$ \\
\hline (Zhou; Xun, 2018) & & & $\mathrm{X}$ & & \\
\hline (Dias et al., 2019) & $\mathrm{X}$ & $\mathrm{X}$ & $\mathrm{X}$ & $\mathrm{X}$ & $\mathrm{X}$ \\
\hline (Galuchi et al., 2019) & & & & $\mathrm{X}$ & \\
\hline (Wolfshorndl et al., 2019) & $\mathrm{X}$ & $\mathrm{X}$ & $\mathrm{X}$ & $\mathrm{X}$ & \\
\hline (Dias et al., 2020) & $\mathrm{X}$ & $\mathrm{X}$ & $\mathrm{X}$ & $\mathrm{X}$ & $\mathrm{X}$ \\
\hline (Jeong et al., 2020) & $\mathrm{X}$ & $\mathrm{X}$ & $\mathrm{X}$ & $\mathrm{X}$ & $\mathrm{X}$ \\
\hline Total & 7 & 9 & 11 & 8 & 5 \\
\hline
\end{tabular}

Fonte: Autores

As cadeias de suprimentos em zonas portuárias do Brasil e China foram investigadas. Jiang, Li e Shen (2018) analisaram a rede empresarial do Qingdao Port Goup na China, enquanto Dias, Leal, de Oliveira (2019) investigaram o Porto de Sepetiba, no Brasil. Os autores buscaram identificar os riscos presentes na cadeia de suprimentos dos portos, perpassando pelos cinco fatores de riscos elencados na Tabela 1. As pesquisas identificaram fragilidades nos sistemas portuários de ambos os territórios, ressaltado em Qingdao o risco de ambiente externo (risco destrutivo, Tabela 1) e no Porto de Sepetiba o atraso de matérias primas por problemas durante o transporte marítimo ou manuseio de cargas (risco de transporte, Tabela 1). 
Os cinco fatores de riscos no SCRM, conforme da Tabela 1, também foram investigados simultaneamente em outros dois estudos, que buscaram identificar e classificar os fatores de riscos, analisando setores distintos: a pesquisa de Dias, Hernandez e Oliveira (2020), no Brasil, é voltada para a indústria automotiva e a pesquisa de Jeong, Cha e Kim (2020), na China, é direcionada ao setor de construção naval. Vale reafirmar que os fatores de riscos adotados por Jeong, Cha e Kim (2020) deram suporte ao presente estudo e estão listados na Tabela 1. Por outro lado, Dias, Hernandez e Oliveira (2020) compilaram fatores de riscos de diferentes bases teóricas, de pesquisas anteriores, adotando como critério seis fatores de riscos: risco de suprimento, risco ambiental, risco de demanda, risco discreto, risco operacional e risco de ruptura. O SCRM na indústria automotiva do Brasil também foi investigado por Wolfshorndl, Vivaldini e Camargo Junior (2019), contudo não foi identificado em seu estudo apontamentos sobre o risco destrutivo.

Conforme a Tabela 1, três artigos revisados neste estudo foram enquadrados nos fatores "risco de informação e previsão" e "risco de fornecedor". Alvarenga et al. (2018), no Brasil, considerou em sua pesquisa a incerteza quanto ao mix de produtos, volume da demanda e a incerteza de fornecimento dos materiais. Concomitante, no contexto China, o estudo de Ji, Qu e Yu (2017) possui como ponto focal a incerteza de demanda na cadeia de suprimentos, enquanto Fan e Stevenson (2018b), buscou analisar os riscos na relação comprador $\mathrm{x}$ fornecedor.

No setor agrícola, Song e Zhuang (2017) e Galuchi, Rosales e Batalha (2019), nos contextos China e Brasil respectivamente, abordaram o risco ambiental nos estudos sobre o SCRM. Enquanto Song e Zhuang (2017) propõe um modelo para controle de aditivos químicos, Galuchi, Rosales e Batalha (2019) estuda a produção de carne bovina e os impactos na região amazônica, introduzindo o conceito de risco reputacional, não identificado nas pesquisas dos demais autores.

\section{CONCLUSÕES}

É essencial o desenvolvimento de pesquisas sobre Supply Chain Risk Management e, para tanto, a declaração PRISMA fornece um terreno fértil para meta-pesquisa (Page ; Moher, 2017). O termo Supply Chain Risk Management é relativamente novo, aparecendo nas bases a partir de: 2003 (Web of Science), 2012 (Scopus) e somente em 2017 (SciELO). As informações apresentadas na seção de resultados poderão auxiliar outros pesquisadores do tema. 
Em número de publicações o SCRM, os contextos Brasil e China estão subrepresentados no cenário internacional de produção de pesquisas, com apenas 6 e 8 artigos elegíveis para revisão, respectivamente, em um total de 103 selecionados. Pesquisadores têm apontado possibilidade de vieses, pesquisas bibliométricas sugerem viés de pesquisadores europeus para Scopus (Elsevier) e viés de pesquisadores Estadunidenses para Web of Science (Clarivate Analytics) (MONGEON; PAUL-HUS, 2016).

As limitações desta pesquisa incluem, evidentemente, a baixa amostragem, contudo não foram identificadas na declaração PRISMA exigência em relação a quantidade mínima de artigos a serem revisados. Além disso, deve ser ressaltado que as diretrizes PRISMA foram publicadas em 2009 e não foram atualizadas desde então (PAGE ; MOHER, 2017), possibilitando questionamentos sobre a necessidade de atualização.

Para os estudos futuros recomenda-se que seja realizada nova revisão sistemática de literatura sobre Supply Chain Risk Management, acrescentado outras bases nas buscas eletrônicas. Espera-se que os resultados aqui apresentados contribuam para que novos pesquisadores do SCRM possam conduzir os seus estudos bibliográficos com maior eficiência e efetividade. 


\section{REFERÊNCIAS}

ALVARENGA, M. Z.; de OLIVEIRA, M. P. V.; FILHO, H. Z.; SANTOS, W. R. Do analytically-oriented supply chains better manage risks? Journal of Operations and Supply Chain Management, v.11, n.2, p.32-45, 2018. https://doi.org/10.12660/joscmv11n2p32-45

BARYANNIS, G.; VALIDI, S.; DANI, S.; ANTONIOU, G. Supply chain risk management and artificial intelligence: state of the art and future research directions. International Journal of Production Research, v.57, n.7, p.2179-2202, 2019. https://doi.org/10.1080/00207543.2018.1530476

CHEN, X.; WAN, N.; WANG, X. Flexibility and coordination in a supply chain with bidirectional option contracts and service requirement. International Journal of Production Economics, v.193, p.183-192, 2019. https://doi.org/10.1016/j.ijpe.2017.07.013

COCHRANE, D. R. R.; MELLO, J. A. V. B. Consequências da economia GIG na identidade profissional: revisão sistemática da literatura. Logeion: Filosofia Da Informação, v.6, n.2, p.142-156, 2020. https://doi.org/10.21728/logeion.2020v6n2.p142-156

CONCOLATO, C. O. F.; CUNHA, M. R.; AFONSO, H. C. A. G. Economic feasibility for photovoltaic solar energy projects: a systematic review. Revista Produção e Desenvolvimento, v.6, 2020. https://doi.org/10.32358/rpd.2020.v6.506

DIAS, G. C.; HERNANDEZ, C. T.; de OLIVEIRA, U. R. Supply chain risk management and risk ranking in the automotive industry. Gestao e Producao, v.27, n.1, p.1-21, 2020. https://doi.org/10.1590/0104-530X3800-20

DIAS, G. C.; LEAL, I. C.; OLIVEIRA, U. R. Supply chain risk management at seaport container terminals. Gestão e Produção, v.26, n.3, 2019. https://doi.org/10.1590/0104530X4900-19

FAGUNDES, M. V. C.; TELES, E. O.; VIEIRA DE MELO, S. A. B.; FREIRES, F. G. M. Decision-making models and support systems for supply chain risk: literature mapping and future research agenda. European Research on Management and Business Economics, v.26, n.2, p.63-70, 2020. https://doi.org/10.1016/j.iedeen.2020.02.001

FAN, Y.; STEVENSON, M. A review of supply chain risk management: definition, theory, and research agenda. International Journal of Physical Distribution \& Logistics Management, v.48, n.3, p.205-230, 2018b. https://doi.org/10.1108/IJPDLM-01-2017-0043

FAN, Y.; STEVENSON, M. Reading on and between the lines: risk identification in collaborative and adversarial buyer-supplier relationships. Supply Chain Management, v.23, n.4, p.351-376, 2018a. https://doi.org/10.1108/SCM-04-2017-0144

GALUCHI, T. P. D.; ROSALES, F. P.; BATALHA, M. O. Management of socioenvironmental factors of reputational risk in the beef supply chain in the Brazilian Amazon region. International Food and Agribusiness Management Review, v.22, n.2, p.155-171, 2019. https://doi.org/10.22434/IFAMR2018.0004 
IVANOV, D.; DOLGUI, A. Low-Certainty-Need (LCN) supply chains: a new perspective in managing disruption risks and resilience. International Journal of Production Research, v.57, n.15-16, p.5119-5136, 2019. https://doi.org/10.1080/00207543.2018.1521025

JEONG, K.; CHA, J.; KIM, Y. SCRM awareness in the shipbuilding and marine equipment market: Empirical evidence from South Korea, China, and Singapore. Sustainability, Switzerland, v.12, n.12, 2020. https://doi.org/10.3390/su12125115

JI, Y.; QU, S.; YU, Z. A New Method for Solving Multiobjective Bilevel Programs. Discrete Dynamics in Nature and Society, 2017. https://doi.org/10.1155/2017/2870420

JIANG, B.; Li, J.; SHEN, S. Supply Chain Risk Assessment and Control of Port Enterprises: Qingdao port as case study. Asian Journal of Shipping and Logistics, v.34, n.3, p.198-208, 2018. https://doi.org/10.1016/j.ajsl.2018.09.003

MOHER, D.; LIBERATI, A.; TETZLAFF, J.; ALTMAN, D. G. The PRISMA Group. Reprint - Preferred reporting items for systematic reviews and meta-analyses: the PRISMA statement. Physical Therapy, v.89, n.9, p.873-880, 2009. PMID: 19723669.

MONGEON, P.; PAUL-HUS, A. The Journal Coverage of Web of Science and Scopus: a Comparative Analysis. Scientometrics, v.106, n.1, p.213-228, 2016.

https://doi.org/10.1007/s11192-015-1765-5

OLIVEIRA, U. R. de; ESPINDOLA, L. S.; MARINS, F. A. S. Analysis of supply chain risk management researches. Gestão e Produção, v.25, n.4, p.671-695, 2018. https://doi.org/10.1590/0104-530X3515-16

PAGE, M. J.; MOHER, D. Evaluations of the uptake and impact of the Preferred Reporting Items for Systematic reviews and Meta-Analyses (PRISMA) Statement and extensions: A scoping review. Systematic Reviews, v.6, n.1, p.1-14, 2017. https://doi.org/10.1186/s13643017-0663-8

PORTER, A. L.; KONGTHON, A.; LU, C. Research profiling: improving the literature review. Scientometrics, v.53, n.3, p.351-370, 2002. DOI: 10.1023/A:1014873029258

PRAKASH, S.; SONI, G.; RATHORE, A.P.S. A Critical Analysis of Supply Chain Risk Management Content: a Structured Literature Review. Journal of Advances in Management Research, v.14, n.1, p.69-90, 2017. DOI: 10.1108/JAMR-10-2015-0073

REQUEIJO, J. F. G.; ABREU, A. J. P. C. F.; CALADO, J. M. F.; DIAS, A. S. M. E. Six sigma business scorecard approach to support maintenance projects in a collaborative context. Revista Produção e Desenvolvimento, v.4, n.1, p.82-97, 2018.

https://doi.org/10.32358/rpd.2018.v4.313

RODRIGUES, A. S. M.; MELlO, J. A. V. B.; AFONSO, H. C. A. G. Desenvolvimiento estimulado por empreendedorismo em incubadoras de empresa: Uma revisão sistemática. Métodos de informacion, v.10, n.19, p.1-27, 2019. https://dx.doi.org/10.5557/IIMEI10-N19001027

SBARDELOTTO, B.; SEVERO, E. A.; GUIMARÃES, J. C. F.; CAPITANIO, R. P. R. Uma exploração bibliométrica das práticas de gestão de projetos e a vantagem competitiva 
sustentável. Revista Produção e Desenvolvimento, v.3, n.2, p.52-64, 2017. https://doi.org/10.32358/rpd.2017.v3.232

SONG, C.; ZHUANG, J. Modeling a Government-Manufacturer-Farmer game for food supply chain risk management. Food Control, v.78, p.443-455, 2017. https://doi.org/10.1016/j.foodcont.2017.02.047

SOUZA, F. L. C.; DANTAS, A. S. Strategies for the evaluation of electrification projects of public transportation by bus. Revista Produção e Desenvolvimento, v.6, 2020. https://doi.org/10.32358/rpd.2020.v6.461

TRKMAN, P.; OLIVEIRA, M. P. V.; Mccormack, K. Value-oriented supply chain risk management: You get what you expect. Industrial Management and Data Systems, v.116, n.5, p.1061-1083, 2016. https://doi.org/10.1108/IMDS-09-2015-0368

WOLFSHORNDL, D. A.; VIVALDINI, M.; CAMARGO JUNIOR, J. B. Hybrid production system: perspectives in supply chain risk management. Revista de Gestão, v.26, n.3, p.313334, 2019. https://doi.org/10.1108/rege-01-2019-0005

WU, D., OLSON, D. L. Supply chain risk, simulation, and vendor selection. International Journal of Production Economics, v.114, n.2, p.646-655, 2008.

https://doi.org/10.1016/j.ijpe.2008.02.013

XAVIER, R. F. Evolução das plataformas de acesso aberto brasileiras: propriedades e perspectivas. Ciência da Informação, v.48, n.3, p.497-500, 2019.

https://www.brapci.inf.br/index.php/res/v/136395\%0Ahttps://www.brapci.inf.br/index.php/res $/ \mathrm{v} / 136395$

ZHOU, K., XUN, H. Intelligent decision method for supplier selection. The Journal of Engineering, 2018, n.16, p.1682-1687, 2018. https://doi.org/10.1049/joe.2018.8321 